\title{
BROTAÇÃO DE GEMAS EM SECÇÕES DE CAULE DE ABACAXIZEIRO 'SMOOTH CAYENNE' TRATADAS COM REGULADORES DE CRESCIMENTO'
}

\author{
RUIMÁRIO INÁCIO COELHO², ALMY JÚNIOR CORDEIRO DE CARVALHO 3 , \\ JOSE TARCISIO LIMA THIEBAUT ${ }^{3}$, JOSÉ CARLOS LOPES ${ }^{2}$
}

RESUMO: Avaliou-se a propagação ex-vitro do abacaxizeiro, 'Smooth Cayenne', utilizando-se de secções de caule tratadas com Ácido Giberélico $\left(\mathrm{GA}_{3}\right)$ e 6-benzilaminopurina (BAP). O delineamento utilizado foi o fatorial fracionado do tipo (1/5) $5^{3}$, com tamanho de secções, $\mathrm{GA}_{3}$ e BAP, e cinco níveis, num total de 25 tratamentos, que consistiram em combinações de secções de diferentes comprimentos $(6 ; 8 ; 10 ; 12 \mathrm{e} 14 \mathrm{~cm})$

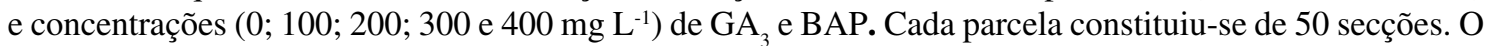
BAP apresentou efeito positivo, e o $\mathrm{GA}_{3}$, efeito negativo sobre a percentagem de secções brotadas em avaliação realizada aos 28 dias após o plantio. $\mathrm{O}$ tamanho da secção, o GA ${ }_{3}$ e o BAP influenciaram no número de gemas brotadas por secção. Verificou-se efeito para as características: altura, massa fresca e massa seca da parte aérea, e massa seca das raízes das mudas avaliadas aos 123 dias após o plantio, em resposta ao tamanho das secções, $\mathrm{GA}_{3}$ e BAP, sendo o efeito positivo apenas para o tamanho das secções.

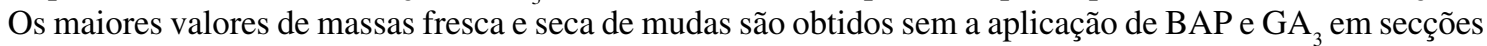
de caule com $14 \mathrm{~cm}$ de comprimento.

Termos para indexação: Ananas comosus var. comosus, propagação, AIB, BAP.

\section{BUD SPROUTING IN STEM SECTIONS OF 'SMOOTH CAYENNE' PINEAPPLE PLANT TREATED WITH GROWTH REGULATORS}

\begin{abstract}
The ex-vitro propagation of the pineapple plant cv Smooth Cayenne was evaluated using the stem sections treated with Giberelic acid $\left(\mathrm{GA}_{3}\right)$ and 6 benzylaminopurine (BAP). The design utilized was the fractionated factorial of the type $(1 / 5) 5^{3}$ with sizes of stem sections, $\mathrm{GA}_{3}, \mathrm{BAP}$ and five levels totalizing 25 treatments that consisted of the combination of different length of section $(6,8,10,12$ and $14 \mathrm{~cm})$ and concentrations $\left(0,100,200,300\right.$ and $\left.400 \mathrm{mg} \mathrm{L}^{-1}\right)$ of GA $\mathrm{G}_{3}$ and BAP. Each plot consisted of 50 sections treated through immersion for one minute in solutions containing different combinations of $\mathrm{GA}_{3}$ e $\mathrm{BAP}$ concentrations. BAP had positive and $\mathrm{GA}_{3}$ negative effect on the percentage of buds sprouting in stem sections at 28 days after planting. The section size, $\mathrm{GA}_{3}$ and $\mathrm{BAP}$ application affected the number of buds sprouting per stem section. It was verified the effect on the following characteristics: shoot height, fresh and dry weight of the seedlings and dry weight of root evaluated 123 days after being planted in answer of the section size, $\mathrm{GA}_{3}$ and BAP. However, only stem section size presented a positive effect. The highest values of fresh and dry weight of the seedlings were obtained without BAP and $\mathrm{GA}_{3}$ in stem sections of 14 cm long.
\end{abstract}

Index terms: Ananas comosus var. comosus, propagation, IBA, BAP.

\section{INTRODUÇÃO}

O abacaxizeiro, tradicionalmente, é propagado usando três tipos de mudas: coroas, rebentões e filhotes. Porém, pode ser multiplicado ex vitro usando-se vários métodos, tais como: seccionamento de caule (Reinhardt \& Cunha, 1981, Giacomelli, 1982), destruição do meristema apical
(Heenkenda, 1993) e pelo tratamento com substâncias do grupo das morfactinas (Adaniya et al., 2004). A propagação in vitro tem sido relatada por vários pesquisadores (Santos Filho et al., 1986; Kiss et al., 1995; Pasqual et al., 1998), porém, no Brasil, a produção comercial ainda é restrita.

Heenkenda (1993) afirmou que a multiplicação rápida empregando secções de caule, em Sri Lanka,

${ }^{1}$ (Trabalho 097-08). Recebido em: 15-04-2008. Aceito para publicação em: 15-11-2008. Parte da Tese de Doutorado do primeiro autor apresentada à UENF. Projeto financiado pela FAPERJ.

${ }^{2}$ Professor, D.Sc., Universidade Federal do Espírito Santo - CCA, Alegre -ES, ruimario@cca.ufes.br, jclopes@cca.ufes.br. ${ }^{3}$ Professor, D.Sc., Universidade Estadual do Norte Fluminense Darcy Ribeiro - CCTA, Campos dos Goytacazes - RJ, almy@uenf.br, thiebaut@uenf.br 
inviabilizou-se devido ao longo tempo requerido para a formação das mudas e da elevada perda verificada no viveiro.

São poucos os relatos sobre o uso de reguladores de crescimento na propagação ex vitro do abacaxizeiro. Suwunnamek(1993), estudando o efeito de Paclobutrazol, Thiourea e Pendimethalin, isolados ou em combinação, sobre a indução de rebentões em abacaxizeiro, verificou que a combinação de $25 \mathrm{mg} \mathrm{L}$ ${ }^{1}$ de paclobutrazol com $500 \mathrm{mg} \mathrm{L}^{-1}$ de thioureia ou $375 \mathrm{mg} \mathrm{L}^{-1}$ de pendimethalin produziu mais rebentões do que isoladamente, tanto a 25 quanto a $50 \mathrm{mg} \mathrm{L}^{-1}$ de concentração.

O tratamento por imersão, durante três horas em solução contendo 2,5 ou $5,0 \mathrm{mg} \mathrm{L}^{-1} \mathrm{de}$ forchlorfenuron ( $N$-(2-chloro-4-pyridyl)- $N$ phenylurea) (CPPU), promoveu a brotação em $85 \%$ das gemas axilares em segmento de caule do abacaxizeiro 'Smooth Cayenne'. E o tratamento com 6-benziladenine, na concentração de 10 ou $25 \mathrm{mg} \mathrm{L}^{-1}$, também foi mais efetivo do que a testemunha (Adaniya et al., 2004).

No Brasil, os únicos relatos sobre estudos relacionados ao emprego destas substâncias, na propagação ex vitro do abacaxizeiro referem-se ao uso de cloroflurenol (Reinhardt \& Cunha, 1999) em pulverização das plantas, poucos dias após a indução artificial do florescimento objetivando a produção de mudas.

Avaliou-se o efeito do ácido giberélico ( $\left.\mathrm{GA}_{3}\right)$, da 6 - Benzilaminopurina (BAP) e do tamanho das secções (TS) sobre a brotação das gemas e o crescimento inicial das mudas na propagação ex vitro do abacaxizeiro 'Smooth Cayenne'.

\section{MATERIAL E MÉTODOS}

O experimento foi instalado na área da Unidade de Apoio à Pesquisa da Universidade Estadual do Norte Fluminense Darcy Ribeiro (UENF), em Campos dos Goytacazes - RJ, situado a $21^{\circ} 48^{\prime}$ de latitude sul e $41^{\circ} 20^{\prime}$ de longitude W.GR., a uma altitude de $11 \mathrm{~m}$ acima do nível do mar, com pluviosidade média anual de $900 \mathrm{~mm}$ e temperatura média de $23,7^{\circ} \mathrm{C}$.

Foram utilizados caules de abacaxizeiro 'Smooth Cayenne' oriundos de uma lavoura comercial estabelecida no município de Campos dos Goytacazes - RJ. A coleta dos caules ocorreu aproximadamente quinze dias após a colheita dos frutos. As plantas selecionadas foram arrancadas e, em seguida, com o auxílio de um facão, tiveram suas folhas eliminadas, com exceção das bainhas.

No galpão, os caules tiveram a parte basal com presenças de raízes, juntamente com o ápice eliminados, utilizando-se de uma guilhotina. Estes então foram seccionados, através de corte transversal ao eixo do talo em pedaços com diferentes comprimentos de acordo com os tratamentos. Em seguida, foram novamente seccionados longitudinalmente obtendo-se quatro secções. Posteriormente, as secções foram tratadas por imersão em solução aquosa contendo benomyl, na concentração de $375 \mathrm{mg} \mathrm{L}^{-1}$, e parathion methyl, na concentração de $0,90 \mathrm{ml} \mathrm{L}^{-1}$, durante 3 minutos, e a seguir colocados para secar à sombra e plantadas no dia seguinte.

O delineamento utilizado foi o fatorial fracionado do tipo $(1 / 5) 5^{3}$, com tamanho de secções, $\mathrm{GA}_{3}$ e BAP, e cinco níveis, num total de 25 tratamentos, que consistiram em combinações de secções de diferentes comprimentos $(6 ; 8 ; 10 ; 12 \mathrm{e}$ $14 \mathrm{~cm}$ ) e concentrações de $0 ; 100 ; 200 ; 300$ e $400 \mathrm{mg}$ $\mathrm{L}^{-1}$ de $\mathrm{GA}_{3}$ e BAP (Conagin \& Jorge, 1982). Cada tratamento constituiu-se de 50 secções, dispostas em 5 fileiras distanciadas $10 \mathrm{~cm}$ entre si e $5 \mathrm{~cm}$ entre secções na fileira, com 24 secções úteis.

No preparo das soluções BAP, antes da diluição em água deionizada, empregou-se o $\mathrm{HCl}$ a $0,1 \mathrm{~N}$ para a dissolução, na proporção de 3 gotas de $\mathrm{HCl}$ para cada $10 \mathrm{mg}$ do BAP, de acordo com recomendação de Caldas et al. (1998). Como fonte do $\mathrm{GA}_{3}$, foi empregado o produto Pro-Gibb ${ }^{\circledR}$, contendo $10 \%$ da substância ativa. $\mathrm{O}$ pH das soluções foi ajustado para 5,6. Para o preparo das soluções, utilizou-se sempre água deionizada.

A aplicação do $\mathrm{GA}_{3}$ e BAP foi realizada aproximadamente 16 horas antes do plantio, por meio de imersão das secções na solução, durante 1 minuto, nas respectivas concentrações, de acordo com os tratamentos. Para isso, as secções foram dispostas em bandejas plásticas na posição horizontal. Toda operação ocorreu a partir das 17 horas, e o plantio das secções iniciou-se às 8 horas do dia seguinte.

As secções foram plantadas na posição horizontal, com as gemas voltadas para cima, em canteiros com $10 \mathrm{~cm}$ de altura e $110 \mathrm{~cm}$ de largura, construídos dentro de uma casa de vegetação, coberta com tela sombrite $30 \%$. O substrato dos canteiros foi preparado utilizando de (v/v) $60 \%$ de terra de superfície, $20 \%$ de areia lavada e $20 \%$ de substrato Plantmax ${ }^{\circledR}$ hortaliça.

Uma semana antes do plantio, foram incorporados $60 \mathrm{~g}$ de superfosfato simples por $\mathrm{m}^{2} \mathrm{de}$ canteiro, e aplicado um herbicida pré-emergente à base de bromacil+diuron, na dose de $2,5 \mathrm{~kg}$ do i.a. por hectare. Os canteiros foram mantidos úmidos por meio de regas periódicas. Foram feitas 
pulverizações quinzenais com fungicida nos primeiros sessenta dias após o plantio.

As avaliações iniciaram-se aos 28 dias após o plantio das secções, com a contagem das secções brotadas, que se repetiu a intervalos semanais, num total de cinco avaliações. Na última avaliação, foram contadas as gemas brotadas por secção, sendo consideradas brotadas as gemas com, pelo menos, $0,5 \mathrm{~cm}$ de altura.

Aos 123 dias após o plantio, as mudas foram colhidas, medidas suas alturas e determinada a massa fresca. As raízes foram lavadas, colocadas em sacolas de papel e, juntamente com a parte aérea, colocadas para secar em estufas de circulação de ar forçada, a $70-75^{\circ} \mathrm{C}$, até peso constante. Após a secagem, foi determinada a massa seca tanto da parte aérea como das raízes.

As análises estatísticas foram efetuadas por meio de análise de regressão. Para as variáveis avaliadas, foi ajustada a superfície de resposta no modelo:

$\mathrm{Y}=\gamma_{0}+\gamma_{1} \mathrm{~S}+\gamma_{2} \mathrm{~S}^{2}+\gamma_{3} \mathrm{G}+\gamma_{4} \mathrm{G}^{2}+\gamma_{5} \mathrm{~B}+\gamma_{6} \mathrm{~B}^{2}$ $+\gamma_{7} \mathrm{SG}+\gamma_{8} \mathrm{SB}+\gamma_{9} \mathrm{~GB}$, onde: $\mathrm{Y}=\mathrm{a}$ variável dependente; $\gamma_{0}$ a $\gamma_{9}=$ coeficientes de regressão; $S=$ tamanho de secções de caule; $\mathrm{G}=$ concentrações de $\mathrm{GA}_{3}$; $\mathrm{B}=$ concentrações de BAP. Com base na significância da regressão, verificada pelo teste F, e da significância dos coeficientes, pelo teste $\mathrm{t}$ de Student, foi considerado um nível aceitável de até $5 \%$ de probabilidade. Os valores dos diferentes níveis das variáveis independentes foram transformados para polinômios ortogonais, utilizando-se dos coeficientes $(-2 ;-1 ; 0 ; 1$ e 2$)$ para componente de $1^{\mathrm{o}}$ grau, e os coeficientes $(2 ;-1 ;-2$; -1 e 2) para componente de $2^{\circ}$ grau.

\section{RESULTADOS E DISCUSSÃO}

A superfície de resposta para percentagem de secções brotadas demonstra o efeito significativo para os componentes lineares de segundo grau do $\mathrm{GA}_{3}\left(\mathrm{G}^{2}\right)$ e de primeiro grau do BAP $(\mathrm{B})$, porém apenas para a avaliação realizada aos 28 dias após o plantio das secções (DAP), sendo o efeito do $\mathrm{GA}_{3}$ depressivo (Tabela 1 e Figura 1A). O maior valor para percentagem de brotação aos $28 \mathrm{DAP}$, estimado pelo modelo, foi de 55,6\%, com secções de $14 \mathrm{~cm} \mathrm{e}$ $400 \mathrm{mg} \mathrm{L}^{-1}$ BAP na ausência de GA (Figura 1A).

A ausência de resposta aos tratamentos para percentagem de secções brotadas aos 35; 42 e 49 dias após o plantio, associada ao comportamento observado aos 28 dias após o plantio, demonstra que o $\mathrm{GA}_{3}$ apenas retardou e que a BAP antecipou o início das brotações. Todavia, não interferiram na percentagem final de secções brotadas (Tabela 1).

Davenporte et al. (2001) relataram que o $\mathrm{GA}_{3}$ endógeno retardou a brotação das gemas em haste decapitada de manga, efeito que acreditam estar associado com o aumento ou manutenção da síntese de auxinas endógenas pelo $\mathrm{GA}_{3}$. Desse modo, o $\mathrm{GA}_{3}$ manteria altas relações auxinas/citocininas, semelhantes àquelas que mantêm dormentes as gemas em outras espécies.

A percentagem de secções brotadas foi a única variável que não apresentou resposta significativa ao tamanho da secção (Tabela 1). Resultado semelhante foi observado por Reinhardt \& Cunha (1981), que também não observaram efeito do tamanho de secções utilizadas sobre o número de secções brotadas.

Observou-se efeito significativo para o tamanho das secções, BAP e GA 3 sobre o número de gemas brotadas por secção (Tabelas 1 e 2). O maior valor para número de gemas brotadas estimado pelo modelo foi de 3,30 com secções de $14 \mathrm{~cm}, 300 \mathrm{mg} \mathrm{L}^{-1}$ $\mathrm{GA}_{3}$ e $400 \mathrm{mg} \mathrm{L}^{-1} \mathrm{BAP}$, contra o menor valor de 2,29 na ausência de $\mathrm{GA}_{3}$ e BAP (Figura 1B). Todavia, apesar do maior número de gemas brotadas (considerando aquelas com altura $>0,5 \mathrm{~cm}$ ), para alguns tratamentos, com avaliação aos 49 dias após o plantio, isto não resultou em maior número de mudas por secção, na avaliação aos 123 dias após o plantio.

$\mathrm{O} \mathrm{GA}_{3}$ promoveu a brotação de várias gemas, que interromperam o crescimento ainda na fase inicial. Considerando que o $\mathrm{GA}_{3}$ atua no alongamento do caule e das folhas pelo estímulo tanto da divisão quanto do alongamento celular (Davies, 1995; Raven et al., 1999), provavelmente, o que ocorreu foi um alongamento das gemas.

Adaniya et al. (2004), estudando o efeito de reguladores de crescimento na propagação do abacaxizeiro 'Smooth Cayenne', verificaram maior eficiência do tratamento com 6-benzyladenine na concentração de 10 e $25 \mathrm{mg} \mathrm{L}^{-1}$ sobre a brotação das gemas (7,6 gemas) em relação ao controle (2,2 gemas), por secção de caule com $12 \mathrm{~cm}$ de comprimento sem corte longitudinal.

$\mathrm{O} \mathrm{GA}_{3}$ promoveu alterações morfológicas nas gemas e nos brotos, na fase inicial do crescimento. Estes se apresentaram mais finos e alongados e com uma curvatura. Nas mudas ainda pequenas, as folhas apresentaram-se muito estreitas em relação àquelas apresentadas pelas mudas das secções que não receberam tratamento com $\mathrm{GA}_{3}$.

As análises dos resultados para a altura das mudas (ALT), massa fresca (MFA) e massa seca (MSA) da parte aérea, aos 150 dias, demonstraram efeitos significativos dos componentes lineares $(\mathrm{S}$, 
G, B) para todos os fatores avaliados, porém estes foram depressivos para $\mathrm{GA}_{3}$ e BAP, indicando redução no crescimento inicial das mudas (Tabela 2 ). Por outro lado, os resultados para tamanho da secção confirmaram o efeito positivo sobre o crescimento inicial das mudas, comportamento semelhante aos observados por outros pesquisadores (Reinhardt \&Cunha, 1981; Braga \& Sá, 2001).

A maior produção de massa fresca e de massa seca estimadas foi de $66,38 \mathrm{~g}$ e $8,49 \mathrm{~g}$, respectivamente, com secções de $14 \mathrm{~cm}$, sem aplicação de $\mathrm{GA}_{3}$ e BAP, porém abaixo do valor médio de 87,6 g obtido por Reinhardt \& Cunha (1981), aos 130 dias após o plantio das secções em sementeira.

A massa seca produzida pelas raízes apresentou comportamento semelhante àqueles verificados para massa fresca (Tabela 2). Ocorreu redução na produção de massa seca das raízes com o aumento nas doses de BAP e de GA. Este resultado pode explicar os efeitos negativos dos tratamentos com $\mathrm{GA}_{3}$ e BAP em relação à produção de massa fresca e massa seca produzidas pelas mudas, uma vez que o crescimento e o desenvolvimento de plantas são dependentes da absorção de água e nutrientes pelas raízes.

Os resultados da massa seca das raízes, em função do tratamento com BAP, estão de acordo com Peres \& Kerbauy (2000), quando afirmaram que as citocininas estimulam a iniciação de gemas do caule, porém inibem a formação de raízes. O maior valor para massa seca das raízes foi estimado em 1,493 g para o tratamento constituído por secções de $14 \mathrm{~cm}$ na ausência de $\mathrm{GA}_{3}$ e BAP (Tabela 2). Os maiores valores para massa seca das raízes foram registrados na ausência de BAP e GA (Tabela 3).

$\mathrm{O}$ efeito negativo apresentado pelo $\mathrm{GA}_{3}$ sobre a massa seca das raízes está em concordância com Meyer et al. (1973), segundo os quais, a formação de raízes em estacas é inibida quando tratadas com giberelinas, inibindo inclusive o efeito auxínico de estímulo à formação de raízes.

TABELA 1 - Percentagem média de secções brotadas do abacaxizeiro 'Smooth Cayenne' e número de gemas brotadas por secção (NGBS), em função do tamanho das secções (S), GA 3 e BAP, e dos dias após o plantio (DAP). UENF, Campos dos Goytacazes (RJ), 2008.

\begin{tabular}{|c|c|c|c|c|c|}
\hline \multirow[b]{2}{*}{ Tratamentos } & \multicolumn{4}{|c|}{ Percentagem de secções brotadas } & \multirow{2}{*}{$\begin{array}{l}\text { NGBS } \\
49 \text { DAP }\end{array}$} \\
\hline & 28 DAP & 35 DAP & 42 DAP & 49 DAP & \\
\hline $\mathrm{S}=6 \mathrm{~cm}$ & 39,14 & 52,06 & 58,36 & 80,70 & 1,21 \\
\hline $\mathrm{S}=8 \mathrm{~cm}$ & 37,48 & 55,36 & 63,36 & 81,46 & 1,44 \\
\hline $\mathrm{S}=10 \mathrm{~cm}$ & 35,84 & 53,34 & 64,16 & 83,68 & 1,84 \\
\hline $\mathrm{S}=12 \mathrm{~cm}$ & 44,04 & 54,16 & 60,02 & 80,62 & 1,83 \\
\hline $\mathrm{S}=14 \mathrm{~cm}$ & 34,88 & 47,38 & 55,00 & 79,56 & 1,94 \\
\hline Média & 38,28 & 52,46 & 60,18 & 81,20 & 1,65 \\
\hline Efeito & ns & ns & ns & ns & $\mathrm{S} * *$ \\
\hline $\mathrm{GA}_{3}=0 \mathrm{mg} \mathrm{L}^{-1}$ & 46,54 & 59,98 & 66,70 & 82,80 & 1,51 \\
\hline $\mathrm{GA}_{3}=100 \mathrm{mg} \mathrm{L}^{-1}$ & 39,16 & 57,90 & 61,66 & 79,84 & 1,64 \\
\hline $\mathrm{GA}_{3}=200 \mathrm{mg} \mathrm{L}^{-1}$ & 31,71 & 51,20 & 60,86 & 82,46 & 1,74 \\
\hline $\mathrm{GA}_{3}=300 \mathrm{mg} \mathrm{L}^{-1}$ & 29,98 & 43,22 & 52,50 & 82,46 & 1,64 \\
\hline $\mathrm{GA}_{3}=400 \mathrm{mg} \mathrm{L}^{-1}$ & 39,04 & 50,00 & 59,18 & 78,46 & 1,69 \\
\hline Média & 37,286 & 52,46 & 60,18 & 81,20 & 1,64 \\
\hline Efeito & $\mathrm{G}^{*} \mathrm{G}^{2} *$ & ns & ns & ns & $\mathrm{G}_{3}{ }^{2} * *$ \\
\hline $\mathrm{BAP}=0 \mathrm{mg} \mathrm{L}^{-1}$ & 33,30 & 44,88 & 53,36 & 79,20 & 1,57 \\
\hline $\mathrm{BAP}=100 \mathrm{mg} \mathrm{L}^{-1}$ & 32,38 & 48,72 & 55,84 & 81,48 & 1,60 \\
\hline $\mathrm{BAP}=200 \mathrm{mg} \mathrm{L}^{-1}$ & 37,52 & 49,16 & 56,72 & 82,30 & 1,63 \\
\hline $\mathrm{BAP}=300 \mathrm{mg} \mathrm{L}^{-1}$ & 35,82 & 55,38 & 65,82 & 76,54 & 1,70 \\
\hline $\mathrm{BAP}=400 \mathrm{mg} \mathrm{L}^{-1}$ & 52,36 & 64,16 & 69,16 & 86,50 & 1,77 \\
\hline Média & 38,28 & 52,46 & 60,18 & 81,20 & 1,65 \\
\hline Efeito & $\mathrm{B} * *$ & ns & ns & ns & $\mathrm{B} * * \mathrm{~B}^{2 *}$ \\
\hline
\end{tabular}

** e * significativo ao nível de 1 e $5 \%$ de prob., pelo teste t de Student. 
TABELA 2 - Superfícies de respostas para altura de muda (ALT), massa fresca (MF) e massa seca (MS) da parte aérea, massa seca das raízes (MSR) de mudas de 'Smooth Cayenne', avaliadas aos 123 dias após o plantio, em função do tamanho de secção de caule (S), do $\mathrm{GA}_{3}(\mathrm{G})$ e BAP (B). UENF, Campos dos Goytacazes (RJ), 2008.

\begin{tabular}{|c|c|c|c|}
\hline Variável & Equações & $\mathbf{R}^{2}$ & $\begin{array}{l}\text { CV } \\
(\%)\end{array}$ \\
\hline $\begin{array}{l}\text { ALT } \\
(\mathrm{cm})\end{array}$ & $\begin{aligned} \mathrm{Y}= & 21,4+2,192 \mathrm{~S}^{* *}-0,992 \mathrm{G}^{* *}- \\
& 0,844 \mathrm{~B}^{*}-0,37 \mathrm{~S}^{2}+0,44 \mathrm{G}^{2}+0,38 \mathrm{~B}^{2}\end{aligned}$ & 0,77 & 20,5 \\
\hline $\begin{array}{l}\text { MF } \\
(\mathrm{g})\end{array}$ & $\begin{aligned} \mathrm{Y}= & 42,7+7,437 \mathrm{~S} * *-4,401 \mathrm{G}^{* *}- \\
& 3,28 \mathrm{~B} * *+0,48 \mathrm{~S}^{2}-0,627 \mathrm{G}^{2}+1,03 \mathrm{~B}^{2}\end{aligned}$ & 0,76 & 45,2 \\
\hline MS (g) & $\begin{aligned} \mathrm{Y}= & 5,35+0,961 \mathrm{~S} * *-0,612 \mathrm{G}^{* *}-0,511 \mathrm{~B} * * \\
& +0,0004 \mathrm{~S}^{2}-0,076 \mathrm{G}^{2}+0,124 \mathrm{~B}^{2}\end{aligned}$ & 0,72 & 47,6 \\
\hline $\begin{array}{l}\text { MSR } \\
(\mathrm{g})\end{array}$ & $\begin{aligned} \mathrm{Y}= & 0,636+0,119 \mathrm{~S}^{* *}-0,107 \mathrm{G}^{*}- \\
& 0,092 \mathrm{~B}^{*}-0,019 \mathrm{~S}^{2}-0,054 \mathrm{G}^{2}+0,05 \mathrm{~B}^{2}\end{aligned}$ & 0,62 & 62,3 \\
\hline
\end{tabular}

** e * significativo ao nível de 1 e $5 \%$ de prob., pelo teste t de Student.

TABELA 3 - Altura (ALT), massa fresca (MF) e massa da matéria seca (MS) da parte aérea e massa da matéria seca das raízes (MSR) das mudas, em função do tamanho das secções (S), tratadas com $\mathrm{GA}_{3}$ (G) e BAP (B), avaliadas aos 123 dias após o plantio. UENF, Campos dos Goytacazes (RJ), 2008.

\begin{tabular}{|c|c|c|c|c|}
\hline \multirow[b]{2}{*}{ Tratamentos } & \multicolumn{4}{|c|}{ Variáveis analisadas } \\
\hline & ALT $(\mathrm{cm})$ & MF (g) & MS (g) & $\operatorname{MSR}(\mathrm{g})$ \\
\hline $\mathrm{S}=6 \mathrm{~cm}$ & 16,3 & 29,2 & 3,525 & 0,390 \\
\hline $\mathrm{S}=8 \mathrm{~cm}$ & 19,6 & 33,2 & 4,097 & 0,557 \\
\hline $\mathrm{S}=10 \mathrm{~cm}$ & 22,0 & 44,0 & 5,648 & 0,694 \\
\hline $\mathrm{S}=12 \mathrm{~cm}$ & 24,1 & 48,2 & 6,083 & 0,817 \\
\hline $\mathrm{S}=14 \mathrm{~cm}$ & 25,0 & 58,9 & 7,331 & 0,855 \\
\hline Efeito & $\mathrm{S} * *$ & $\mathrm{~S} * *$ & $\mathrm{~S} * *$ & $\mathrm{~S} * *$ \\
\hline $\mathrm{GA}_{3}=0 \mathrm{mg} \mathrm{L}^{-1}$ & 24,04 & 49,52 & 6,298 & 0,985 \\
\hline $\mathrm{GA}_{3}=100 \mathrm{mg} \mathrm{L}^{-1}$ & 22,08 & 48,80 & 6,187 & 0,670 \\
\hline $\mathrm{GA}_{3}=200 \mathrm{mg} \mathrm{L}^{-1}$ & 21,30 & 43,44 & 5,755 & 0,696 \\
\hline $\mathrm{GA}_{3}=300 \mathrm{mg} \mathrm{L}^{-1}$ & 18,72 & 37,98 & 4,226 & 0,359 \\
\hline $\mathrm{GA}_{3}=400 \mathrm{mg} \mathrm{L}^{-1}$ & 20,76 & 33,70 & 4,174 & 0,603 \\
\hline Efeito & $\mathrm{G}_{3} * *$ & $\mathrm{G}_{3} * *$ & $\mathrm{G}_{3} * *$ & $\mathrm{G}_{3} * *$ \\
\hline $\mathrm{BAP}=0 \mathrm{mg} \mathrm{L}^{-1}$ & 23,36 & 51,20 & 6,660 & 0,930 \\
\hline $\mathrm{BAP}=100 \mathrm{mg} \mathrm{L}^{-1}$ & 22,86 & 43,95 & 5,491 & 0,688 \\
\hline $\mathrm{BAP}=200 \mathrm{mg} \mathrm{L}^{-1}$ & 20,36 & 44,16 & 5,559 & 0,576 \\
\hline $\mathrm{BAP}=300 \mathrm{mg} \mathrm{L}^{-1}$ & 19,50 & 34,60 & 4,338 & 0,475 \\
\hline $\mathrm{BAP}=400 \mathrm{mg} \mathrm{L}^{-1}$ & 20,82 & 39,52 & 4,600 & 0,644 \\
\hline Efeito & $\mathrm{B}^{*}$ & $\mathrm{~B} * *$ & $\mathrm{~B} * *$ & $\mathrm{~B}^{*}$ \\
\hline
\end{tabular}

** e * significativo ao nível de 1 e $5 \%$ de prob., pelo teste $\mathrm{t}$ de Student. 

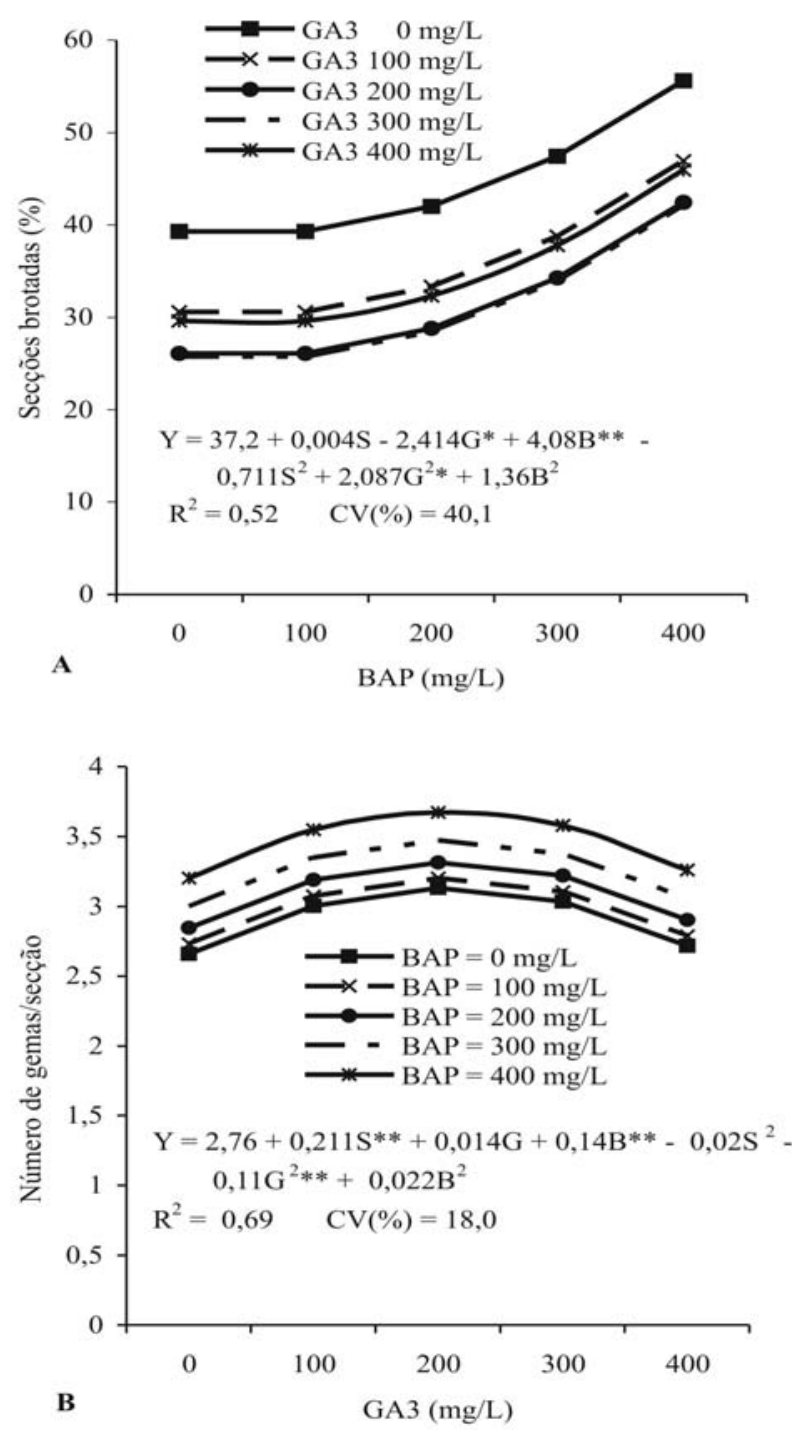

FIGURA 1 - Porcentagem de secções brotadas aos 28 dias após o plantio (A) e Número de gemas brotadas por secção aos 49 dias após o plantio (B), estimados pela superfície de resposta, em função de $\mathrm{GA}_{3}$ e BAP, para secções com $14 \mathrm{~cm}$ de comprimento. UENF, Campos dos Goytacazes (RJ), 2008.

\section{CONCLUSÕES}

1-O tratamento das secções com os fitorreguladores $\mathrm{GA}_{3}$ e BAP não influencia na produção de mudas do abacaxizeiro cv. Smooth Cayenne.

2 - Os maiores valores para massa da matéria fresca e massa da matéria seca das mudas são obtidos sem aplicação de BAP e GA, utilizando-se de secções de caule com $14 \mathrm{~cm}$ de comprimento.

\section{REFERÊNCIAS}

ADANIYA, S.; MINEMOTA, K.; MOROMIZATO, Z.; MOLOMURA, K. The use of CPPU for efficient propagation of pineapple. Scientia Horticultura, Amsterdan, v.100, p.7-14, 2004.

BRAGA, M.F.; SÁ, M.E.L. de. Smooth Cayenne pineapple propagation by stem sections. Revista Brasileira de Fruticultura, Jaboticabal, v.23, n.1, p.175-178, 2001. 
CALDAS, L.S.; HARIDOSAN, P.; FERREIRA, M.E. Meios nutritivos. In: TORRES, A.C.; CALDAS, L.S.; BUSO, J.A. (Ed.). Cultura de tecidos e transformação genética de plantas. Brasília: EMBRAPA-SPI, 1998. p.371-394.

CONAGIN, A.; JORGE, J. de P.N. Delineamento (1/5) $(5 \times 5 \times 5)$ em Blocos. Bragantia, Campinas, v.41, p.155$168,1982$.

DAVENPORTE, L.D.; PEARCE, D.W.; Rood, S.B. Correlation of endogenous Gibberellic Acid with iniciation of Mango shoot growth. Journal Plant Growth Regulator, New York, v.20, p.308-315, 2001.

DAVIES, P.J. (Ed.). Plant hormones: physiology, biochemistry and molecular biology. $2^{\text {nd }}$. ed. London: Kluwer Academic Publishers, 1995. 833p.

GIACOMELLI, E.J. Expansão da abacaxicultura no Brasil. Campinas: Fundação Cargill, 1982. 79p.

HEENKENDA, H. M. S. Effect of plant size on sucker promotion in 'Mauritius' pineapple by mechanical decapitation. Acta Horticulture, Wageningen, v.334, p.331-336, 1993.

KISS, E.; KISS, J.; GYULAI, G.; HESZKY,L.E.Amovel method for rapid micropropagation of peneapples. Hortscience, Alexandria, v.30, n.1, p.127-129, 1995.

MEYER, B.; ANDERSON, D.; BÖHNING, R.; FRATIANNE, D. Introdução à fisiologia vegetal. 2.ed. Lisboa: Fundação Colouste Gulbeukian, 1973. $710 \mathrm{p}$.
PASQUAL, M.; MOREIRA, M.A.; SOBRINHO, A. dos A. Biotecnologia aplicada à produção de mudas de abacaxi. Informe Agropecuário, Belo Horizonte, v.19, n.195, p. 20-23, 1998.

PERES, L.E.P.; KERBAUY, G.B. Controle hormonal do desenvolvimento das raízes. Universa, Brasília, v.8, p.181-195, 2000.

RAVEN, P.H.; EVERT, R.E.; EICHHORN, S.E. Fisiologia vegetal. 6.ed. Washington: W.H. Freeman and Company,1999. 906p.

REINHARDT, H.R.C.; CUNHA, G.A.P. da. Métodos de programação. In: CABRAL, J.R.S.; SOUZA, L.F.S. O abacaxizeiro: cultivo, agroindústria e economia. Brasília: Embrapa Mandioca e Fruticultura, 1999. p.105-138.

REINHARDT, D.H.R.C.; CUNHA, G.A.P. da. Método de produção de mudas de abacaxi livres de fusariose I. Comportamento de sementeira e viveiro. In: CONGRESSO BRASILEIRO DE FRUTICULTURA, 6., 1981. Recife. Anais... Recife: SBF, 1981. p.173-192.

SANTOS FILHO, H.P.; DANTAS, J.L.L.; CABRAL, J.R.S. Programa de pesquisa com biotecnologia do Centro Nacional de Pesquisa de Mandioca e Fruticultura. Revista Brasileira de Fruticultura, Jaboticabal, , v.8, n.3, p.23-37, 1986.

SUWUNNAMEK, U. Effect of paclobutrazol, thiourea, and pendimethalin alone or in combination on the induction of suckering in pineapple. Acta Horticulturae, Wageningen, v.334, p.247-254, 1993. 